\title{
Utilización de aminoglicósidos en un hospital pediátrico
}

\author{
Inés Ruiz A. ${ }^{1}$; Marcela Pezzani V. ${ }^{1}$; Avelina Miranda O. ${ }^{2}$ \\ Utilization of aminoglycoside antibiotics \\ in a pediatric hospital
}

\begin{abstract}
Drug utilization review (DUR) methods and the degree of compliance to focal standards for antibiotic therapy were used to asses adequacy of indications for treatment with aminoglvcoside antimicrobials in intants and children from a metropolitan pediatric hospital at Santiago, Chile. One hundred patients who had been thus treated (amikacin 92 cases, gentamicin 8 patients) from june throughout august 1990 were included in the study. In $84.0 \%$ of cases therapy had rational basis, including all three treatments which were grounded on microbiological findings. These criteria should be eventually applied to evaluate appropriate use of other antimicrobial therapies.
\end{abstract}

(Key words: Aminogivcosides, antibiotics, therapy, uses, therapeutics committee, infants and children.)

Los aminoglicósidos son antibióticos ampliamente utilizados en el tratamiento de infecciones causadas por gérmenes gram negativos en pacientes pediátricos. Estos antibióticos presentan estrecho margen terapéutico, alta variabilidad interindividual de las caracteristicas farmacocinéticas y gran incidencia de reacciones adversas (nefrotoxicidad y ototoxicidad), especialmente en pacientes de edades extremas y en quienes existe una falla renal previa ${ }^{1-4}$.

Este estudio fue diseñado con el propósito de revisar la utilización de los aminoglicósidos más empleados en nuestro medio (amikacina, gentamicina), en pacien tes pediátricos, aplicando el método de los estudios de revisión de la utilización (DUR) ${ }^{5}$, que permiten establecer cómo $\mathrm{y}$ en quiénes se utilizan los medicamentos, centrándose específicamente en el paciente, su objetivo principal es la prescripción racional de los

1. Químico-Farmacéutico. Asignatura de Farmacia Clinica. Departamento de Ciencias y Tecnología Farmacéutica, Facultad de Clencias Químicas y Far. macéuticas, Universidad de Chile.

2. Quúmico-Farmacéutico. Servicio de Farmacia, Hospital Roberto del Río (SSNM). fármacos, con la consiguiente mejoría de la calidad de la terapia, lo que implica a su vez disminución de los $\operatorname{costos}^{6-8}$.

\section{Pacientes y Métodos}

El estudio se llevó a cabo en el Servicio de Pediatría del Hospital Roberto del Río, perteneciente al Servicio de Salud Metropolitano Norte đel Sistema Nacional de Servicios de Salud, que consta de 360 camas pediátricas, durante los meses de junio a agosto de 1990. Se eligió este servicio por ser el más representativo det consumo de aminoglicósidos en ese establecimiento hospitalario.

Diariamente, utilizando un sistema de alarma, se identificaron las recetas de los pacientes que recibian tratamientos con amikacina y gentamicina. Una yez identificado el paciente se realizó un seguimjento prospectivo de cada una de las terapias, obteniendo los parámetros para cada evaluación de las respectivas historias clínicas. La información obtenida se registró en fichas especialmente diseñadas para el estudio.

Cada una de las indicaciones del aminoglicósido se evaluó de acuetdo a su concordancia con las normas terapéuticas del hospital, considerando las causas de la indicación, información disponible sobre el paciente (edad, peso, sexo, diagnóstico, función renal), las ca. racter ísticas de la administración del antibiótico (vía, dosificación, intervalo y duración de le terapia), los parámetros de eficacia, las causas de la suspensión o cambio de la terapia, las asocinciones de antimicrobianos, las complicaciones y la efectividad de ella. 
Para este estudio los pacientes fuerom estratificados de acuerdo al rango de la edad en neonatos, entre 0 y 29 días; lactantes, cntre uno y 24 ineses; preescolares, entre dos y cinco años; escolares mayores de 5 y menores de 12 años; adolescentes, entre 12 y 15 años.

En to que respecta a los futidamentos de su clección, las terapias fueron clasificadas en empíricas y no empúricas. Dentro de las primeras se agruparon aquellas en que se comenzó a utilizar aminoglicósidos en base a signos $y$ síntomas de infección, desconocléndose el resultado del cukivo cotrespondiente. Los casos en que se había identificado el germen causante de la infección o se conocía el resultado del antibiograma, antes de comenzar los tratamjentos antibióticos, se consideraron terapias no empíricas. Los tratamientos profilácticos no fueron evaluados.

Las terapias empiricas se consideraron clínicamente justificadas de acuerdo a las normas terapéuticas internas del hospital, en los recién nacidos con infección grave; en nifios con síndrome febril y neutropenia; en pacjentes con sindtome diarreico agudo o won bronconeumonias, ambos nosocomiales. Estas terapias se evaluaron de acuerdo a la confirmación del diagnóstico por exámenes y procedimientos de diagnóstico (hemograma, proteína $\mathrm{C}$ reactiva, eultivo $\mathrm{y}$, cuando correspondió, radiografía de tórax). Una vez cvaluadas las razones de la indicación de la terapia se consideraron las siguientes indicadores de adecuación en el uso: ajuste posológico para cada paciente, la duración del tratamiento y los controles realizados al tinal de la tesapia.

Se estimó que la duración de la terapia era adecuada cuando se extendió entre 7 y 10 días. Asimismo se est $j-$ mó inadecuada si se mantuvo a pesar de haberse comprobado, posteriormente, resistencia al antibiótico elegido o aislado un germen para el cual existía otro medicamento de primera elección o siendo los síntomas, en opinión del médico, más sugerentes de infección yiral. Se consideró que existió control apropiado, al término de la tarapia, cuando se efectuaron a lo menos dos de las siguientes determinaciones: temperatura, hemograma, cultivos del foco de la infección, radiografía de tórax y proteína $C$ reactiva cuando corres. pond ía.

Las terapias estudiadas se estandarizaron en diferentes categorías de justificación buena o escasa, de acuerde al cumplimiento de los siguientes criterios previamente establecidos: Se consideró que la terapia estaba bien justificada y se la calificó en grado I, cuando había fundamentos clínicos o bacteriológicos 0 ambos, la dosificación se realizó de acuerdo a las características del paciente, el tratamiento tuvo una duración adecuada $y$ se realizaron controles al finalizarlo; bien justificada en grado II, cuando la duración del tratamiento fue menor a 7 días o superior a 10 dias, pero estaba debidamente fundamentada en las normas terapéuticas, a se suspendió porque exist ía otro de primera elección, o se confirmó la etiolog ja viral. Se consideró terapia con buena justificación en grado III, cuando se suspendió el tratamiento por no hăberse confirmado el diagnóstico inicial o se determinó su inefectividad. La terapia de justificación escasa, por su parte, fue calificada como grado 1 cuando se inicíó sin tomar en cuenta criterios clínicos, bacteriológicos o ambos; grado II cuando no se encontró justificación cfítica bacterioiógica para la duración inadecuada del tratamiento; grado JII si se empleó un régimen inadocuado de dosificación.

\section{Resultados}

Durante el periodo del estudio se evaluaron 100 terapias con aminoglicósidos (amikacina y gentamicina), que representaron $14,2 \%$ del total de las terapias con antibióticos en el servicio de pediatría del hospital. De éstas, 92,0\% correspondieron a amikacina y el resto a gentamicina.

Del total de los casos evaluados, $75 \%$ se dieron en niños de sexo masculino; $38,0 \%$ en neonatos, $55,0 \%$ en lactantes, $5,0 \%$ en preescolares y $2,0 \%$ en niños entre 5 y 15 años de edad. Las unidades que indicaron mayor número de tratamientos fueron recién nacidos $(37,0 \%)$ y lactantes $(35,9 \%)$, mientras infecciosos y medicina registraron menor número de indicaciones con 3,9 y $2,9 \%$, respectivamente. Noventa y siete de los 100 tratamientos con aminoglicósido (89 con amikacina) fueron calificados como empíricos. Los tres restantes, no empíricos, correspondieron todos a amikacina.

En los 100 pacientes incluidos en el estudio se registraron 104 diagnósticos principales, que correspondieron a bronconeumonia (n: 46), sindrome diarreico agudo (n: 22), enfermedad bronquial obstructiva crónica (EBOC) (n: 8), los demás correspondían a infecciones en pacientes inmunodeprimidos (n: 7 ), sepsis neonatal (n: 7), infecciones del tracto urinario y endocarditis bacteriana ( $n: 14)$.

En 84 casos las terapias con aminoglicósidos fueron calificadas en alguno de los grados de buena justificación, mientras los 16 restantes lo fueron en algún grado de justificación escasa. Por otra parte, $58,0 \%$ de las bien justificadas fueron clasificadas en el grado I y $88,0 \%$ de las escasamente justificadas lo fueron en su grado II, destacando que sólo 6 de las 16 terapias menos justificadas correspondian al grado lll (tabla l).

Al relacionar la terapia empleada con el nivel de información microbiológica disponible al hacer la elección injcial, los tres tratamientos no empíricos -como era de esperar-fueron evaluados en algún grado de buena justificación, mientras en los 97 tratamientos empíricos ésta se encontró en 81 casos $(87,5 \%)$.

En $91,0 \%$ de los casos el aminoglicósido se empleó en asociación con uno o más antibió- 
Tabla 1

Distribución de cien terapias con aminoglicósidos en riños según sus grados de justificación

\begin{tabular}{|c|c|c|c|c|c|c|}
\hline & & & & & & \\
\hline & $\mathrm{n}$ & $\%$ & $\pi$ & $\%$ & $n$ & $\%$ \\
\hline $\begin{array}{l}\text { Buena } \\
\text { justificaci }\end{array}$ & & & & & & \\
\hline $\begin{array}{l}\text { Grado I } \\
\text { Grado II } \\
\text { Grado III }\end{array}$ & $\begin{array}{r}47 \\
30 \\
3\end{array}$ & $\begin{array}{l}96,0 \\
97,0 \\
75,0\end{array}$ & $\begin{array}{l}2 \\
1 \\
1\end{array}$ & $\begin{array}{r}4,0 \\
3,0 \\
25,0\end{array}$ & $\begin{array}{r}49 \\
31 \\
4\end{array}$ & $\begin{array}{r}58,0 \\
37,0 \\
5,0\end{array}$ \\
\hline Total & 80 & 95,2 & 4 & 4,8 & 84 & 100,0 \\
\hline $\begin{array}{l}\text { Escasa } \\
\text { justificaci }\end{array}$ & & & & & & \\
\hline $\begin{array}{l}\text { Grado I } \\
\text { Grado II } \\
\text { Grado III }\end{array}$ & $1 \overline{1}$ & $\begin{array}{r}78, \overline{6} \\
100,0\end{array}$ & $\begin{array}{l}1 \\
3 \\
-\end{array}$ & $\begin{array}{r}100,0 \\
21,4 \\
-\end{array}$ & $\begin{array}{r}1 \\
14 \\
1\end{array}$ & $\begin{array}{r}6,0 \\
88,0 \\
6,0\end{array}$ \\
\hline Total & 12 & 75,0 & 4 & 25,0 & 16 & 100,0 \\
\hline
\end{tabular}

ticos de otro tipo, lo que ocurrió siempre que se usó gentamicina (tabla 2). Los antibióticos asociados fueron penícilinas en $78 \%$ de los casos, en $16 \%$ cefalosporinas $y$ en $6 \%$ cotrimoxazol, cloramfenicol o metronidazol.

\section{Comentario}

A pesar de que los efectos nefrotóxicos y ototóxicos de los aminoglicósidos han sido ampliamente estudiados en adultos, para quienes se han desarrollado numerosas sugerencias que promueven su correcta utlización ${ }^{3}$, en los pacientes pediátricos la información disponible es escasa. Debido a que estos medicamentos son ampliamente utilizados para tratar infecciones nosocomiales, especialmente en recién nacidos y lactantes, el presente estudio representa una primera aproximación a la evaluación del uso de aminoglicósidos en hospitales pediátricos chilenos.

El análisis de decisiones terapéuticas utilizando criterios previamente establecidos proporcionó la información necesaria para asignar grados de justificación a los tratamientos así evaluados y determinar las distintas modalidades en el uso de estos agentes. En el futuro se podría
Tabla 2

Frecuencia de asociación de aminoglicósidos con otros antibióticos en 100 casos de tratamientos en aifios

\begin{tabular}{lccc}
\hline Terapia & $\begin{array}{c}\text { Amikacina } \\
\mathbf{n}\end{array}$ & $\begin{array}{c}\text { Gentamicina } \\
\mathbf{n}\end{array}$ & $\begin{array}{c}\text { Total } \\
\%\end{array}$ \\
\hline Monoterapia & 9 & 0 & 9 \\
Biasociada & 65 & 8 & 73 \\
Triasociada & 16 & 0 & 16 \\
$>$ tres & 2 & 0 & 2 \\
\hline Total & 92 & 8 & 100 \\
\hline
\end{tabular}

proyectar el empleo de tablas de decisiones para analizar la utilización de antimicrobianos en los diferentes servicios del hospital. Para esto sería necesario contar con el apoyo del Comité de Farmacia y Terapéutica en la revisión de la utilización y de esta manera minimizar los efectos adversos y optimizar los recursos económicos.

A pesar que $84 \%$ de las terapias evaluadas se consideró con algún grado de fundamento, la gran mayoria de ellas correspondio a terapias empíricas cuya justificación fue netamente 
clínica, sin parámetros objetivos ni cuantificables. Una posible solución sería contar con un profesional experto en uso de antimicrobianos, que pudiese discutir las justificaciones clînicas y aplicar la tabla de decisiones.

En la mayor parte de los casos, la posología y frecuencia de administración suelen calcularse de acuerdo al peso total del paciente, con poca consideración a otros factores que pueden afectar la disponibilidad de los medicamentos, especialmente en los recién nacidos ${ }^{10}$. El ajuste ideal de dosis debería considerar la edad del paciente, la maduración de sus órganos de metabolización y excreción del medicamento, como también - -lo que en nuestro medio no siempre es posible-sus concentraciones plasmáticas ${ }^{11}$.

La principal causa para calificar de escasamente justificado un tratamiento fue la duración inadecuada del mismo, lo que frecuentemente se debió a fallas de coordinación del equipo de salud, en que, una vez que el médico indicó la suspensión de la terapia, esto se cumplió con atrasos de 24 y en algunos casos hasta 72 horas, no ocurriendo lo mismo con su iniciación, que era inmediata.

La medición de la efectividad clínica de los tratamientos con aminoglicósidos excedia las posibilidades del estudio, ya que en la mayoría de los casos éstos se asociaron a otros antimicrobianos, lo que no permitía evaluar realmente cuál fue su papel por separado, sin estudiar cada asociación con un método apropiado.

las definiciones que hemos empleado en esta investigación podrían ser de utilidad para evaluar éstas y otras indicaciones en términos de justificación y efectividad con miras a reducir los riesgos y costes de los tratamientos.

\section{Resumen}

Con el propósito de analizar la racionalidad de la utilización de amikacina y gentamicina en un servicio de pediatría de un hospital metropolitano de niños, se analizaron 100 casos de tratamientos con estos aminoglicósidos, los que fueron realizados durante los meses de junio a agosto de 1991, mediante técnicas de revisión de la utilización (DUR) y definiciones del grado de justificación de las terapias. Noventa y dos pacientes recibieron amikacina y ocho gentami. cina. En $84,0 \%$ de las terapias había buena justificación para usar aminoglicósidos, incluyendo todos los casos en que éstos se prescribieron sobre la base de cultivos, con o sin antibiogramas, mientras en los $16 \%$ restantes ella era escasa o inexistente. Esta metodologia podría ser aplicada al uso de otros antimicrobianos de manera de poder evaluar periódicamente la justificación de las correspondientes terapias.

(Palabras claves: Antibiótico, aminoglicósidos, usos terapéuticos, normas, comité de terapia, comité de farmacia.)

\section{Referencias}

1. Hasletl T, Reynol LR: Aminoglicoside utilization. Am J Hosp Pharm 1988; 23: 872-880.

2. Gonzólez Martin $G$, Bravo $I$, Vargas H, Arancibia A: Pharmacokinetics of gentamicin in children with nephrotic syndrome. Int J Clin Pharmacol Ther Toxicol 1986: $24: 555-558$.

3. Llumes D: Aminoglicaside nephrotoxicity. Kidney International. 1988; 33: 900-911.

4. Detorres $Q$ y white $R E$. Effect of aminoglicoside -Use restriction on drug cost. Am J Hosp Pharm 1984; $41: 1137-1139$.

5. Sarmienio D, Ruiz $I$, Talhouk L, Pezzani $M$ : Utilización de antimicrobianos de alto costo en el Hospital Roberto del Río durante el período 1981-1989. Tesis para optar al título de Químico Farmacéutico. Facultad de Ciencias Químicas y Farmacéuticas. Universidad de Chile. 1989.

6. Ice $D$ and Bergman $U$ : Studies of drug utilization in: BL Strom Pharmacoepidemiology, Nueva York: Editorial Churchill, $1989 ; 259-274$.

7. Hernindez $P$, Davidson $E$, Steven $F$, Baurens $C$ and Chamberlain $T$ : Drug use evaluation, part I: Concepts and methods for Evaluating the use of Drugs in organized health Care Environments. The Consultant Pharmacist 1991;6: 643-648.

8. Feinberg $L$ : Meeting the mandate for anality assurance through drug evaluation. The Consultant Pharmacist. 1991;6:611-618.

9. Aguilo $C$, Emparanzo $E$, Rizzardini $M$ : Normas de atención pediátrica. Hospital Roberto del Río, tercera edición. Santiago: Editorial Mediterráneo, 1989.

10. Kearns $G$, Reed DM: Clinical pharmacokinetics in infants and children. $A$. Reappraisal. Clin Pharmacokinet 1989;17: (supl 1): 29-67.

11. Ases $L$ : Elementos de referencia para la utilización correcta de medicamentos en neonatología. Revista AEFH 1982; 1 ; 233-241. 